\section{Der Schraubenstreckverband.}

Von Dr. H. Töpfer, Stabsarzt der Res. u. Chefarzt einer San.-Komp.

Für die Hcilung der inı Kriege so hä̉ufigelı Knochenbrüche und für die spätere Gebrauchsfähigkeit der verletzten Glieder ist die Festlegung der gebrochenen Gliedmaßen und die gute Einstcllung der Knoche' 1 stücke von größter Wichtigkeit. Zur Erreichung diescer Zicle gibt es mohrere Methoden, deren Anwenduıg natürlich von del Erfahrung und Uebung des behandelnden Arztes, von den äußeren Verhältnissen ind dem. zur Verfügung stehenden Verbandmatcrial abhängt. Allc blutigen Verfahren zur Aneinanderlegung der Knochenstücke, wie die Knochennaht mit Draht, das Zusammenlaalten der Bruchenden durcl Klammern nach Gussenbauer oder Stahlbandplatten nit Ver'schrau bung nach Lane, ferner die Nagelextension sind im Felde nicht anzuwenden und auch in der Friedenspraxis bis auf wenige Fälle ent bchrlicl.

Besteht keinc wesentliche Verschiebung der Bruclistiicke, so ' $\mathrm{r}$ reicht man die Heilung mit dem cinfachen festliegenden Verbande mittels Schienen, Papphulsen oder am bestcil init dem gefensterten odcr unterbrochenen, mit Bügeln versehenen Gipsverbaud. Wenn aber die Bruchflächen der Knochen sich nicht gegenubbrstehen, wie besonders bei Oberschenkelbruchen, infolge des Zusamnienzicheis der starken Muskulatur, so muß man durch halbe Beugung des Gliedes und durch Zug der Muskelkraft entgegenwirken und dadurclı die Bruchstücke möglichst aufeinander einstellen. In dell meiste'll Fảlle'n gibt híerfür der bekannte Streckverband naoh Bardenhcuer vollkonımell befriedigende Resultate. Natürlich kann dieger nur danı angelegt werden, wenn der Verletzte längere Zeit, anı besten bis zur Heilung, all einem Orte bleibt. Da für den Transport dieser Verband ungecignet ist, so habe ich besonders für Kriegszwecke einen tralisportablen Streckverband angegeben ${ }^{1}$ ), del mit einfachen vorhandenen Mitteln leicht anzulegen ist und der Zug und Gegenzug in sich selbst enthält.

Iäßt sich dic Bruchstückverschiebung schon durch cinen kläfligr'lı Zug an dem vorletzten Gliede sofort wieder ausgleichen, so kallu nıan den Strcckgipsverband anlegen. Für diesen Zweck habe ich zun Gebrauch im Felde eine Streckvorrichtung ${ }^{2}$ ) angegeben, die sicl aus eincr verstärkten Kramcrschiene oder cincnı starkell, reclitwinklig gebogenen Bandeisen leicht herstellen und an jedem Operationstiscli befestigen läßt. In diese läßt sich das gebrochene Glied einspannen, kann bis Ausgleich der Knochenverschiebung gestreckt und eingegipst werden.

Das technisch Vollkommenste auf dein Gebiete des Streckverbandes ist durch' die Hackenbruchschen Distraktionsklanmern gelicfert worden. Sie gestatten cinen guten Zugang zur Wunde, und durch allulähliches Nachstellen der Schrauben kann, natürlich unter Rontgenkontrolle, die Streckung soweit fortgesetzt werden. bis dic Bruchenden sich möglichst gegenüberstehen.

Bei dem hohen Preis der Klammern können dicse natilulich niclit fiil' den Gebrauch im Felde zur Verfilgung gestellt wcrden, zuntal ihrc Anlegung eine gewisse Uebung erfordert und daher nicht von jedenı Arzt verlaingt welden kann.

Un die Vorzüge der Hackenbruchschen Klanmorn nicht $\% u$ elitbchren, bill ich bestrebt gewesen, cinell cillfachen Ersatz fur sie zu schaffen.

In jedem Eisengeschaft in Frankieich labe ieh 20 em lange Bolzensclirauben nit Muttern uefunden, wic sie in Bilde la dargestellt silıd. Zu diesclı habe ich"10 cul lange Hilsen odel Muffen von cinem Hehnicd

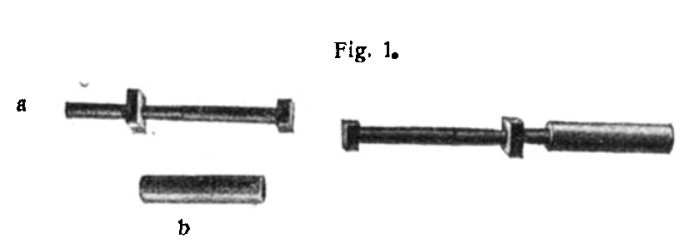
anis Bandeis'n herstellen lassen (s. Fig. 1 b; Schraube mit anfgesterkter Hiilse in e). Damit die Schraube und die Hulse forst cingegipst cine Ende der Muffe breit sehmieden und mubiegen lasse'n.

Die Anlegung des Velbandes goht dann folgendermaßcil vor sich. Wenı es sich $1 \mathrm{~m}$ einell Schußbruch handelt, wird zunächst dic Wunde versorgt. Dann wird denı gebrochenen Gliede eine möglıchst gute Stellung gegeben, bei Armbrüchen durch Bindenzingel, bei Obcroder Unterschenkelbl'iehen spanne ich das Glied in clie von nir aligege be'ne Streckvorrichtulng ('in. Diesc bietet außer de' Sitreckung den Vorteil,

1) D, m, W. $1916 \mathrm{Ni}, 2$ u. 8, - $\left.{ }^{2}\right)$ D. $111, W, 1916 \mathrm{~N} 1,18$ daß clas Glied unabhällgig von Mc'lschenhand ruhig in jedev gewünschten Stellung gelialten wi'd. Nun werden außcr der üblichen Wattepolsterung besonders die Teile mit Wattckissen bedeckt, die dem starken Gegendruck ausgesetzt sind, beim Arm dic Achsclhöhle und die Ellenbeuger (Handgelenk), bcim Ober- und Unterschenkel Rollhügel, Sitzknorren. Kniegegend, Fußrucken und Fersc, Die Gcgend der Bruchstellc wird natürlich nur mit Gaze und Binde zur Bcdeckung der Wunde versehen, die später so oft wic nötig verbunden werden kann. Ober- und unterhalb dieser ungefăhr handbreiten Stelle werden Gipsbinden in der üblichen Weise angelegt, und die Schrauben nit den Hiilsen werden cingegipst. Den fertigen Verband bei einem einfachen Bruch des Oberschenkcls an der Grenze des oberen und mittleren Drittels mit einer Wunde am Kuie zeigt Fig. 2 Zur besserell

Veranschaulichung moge die schematische Zeichuung in Fig. 2 dienen.

$$
\text { In ähnlich' } \mathrm{l}^{\prime}
$$

Weise kann der

Verband auch am Al'in angclegt werdell. Bei Anleguing des Verbandes sind die Muttern möglichst weit auf das Gewinde aufgedreht. Werden nun nuit einem lcicht herzustcllenden Schraubensehlïissel die Muttern wieder abgedrcht, so drücken sie gegen die fest eingegipsten Hülsen, drängen mit diesen den distaleı Teil des Gipsverbandes vom proximalen fort und ziehen so, da der Gipsverband den Körperteilen fest anliegt, aucl dic Bruchstucke anseinander. Tảglich können die Schrauben, ohn daß dem Verlctzton schmerzen entstchell, angezogen werden, bis dic Röntgenkontrolle pine gute Einstellung der Knochenenden ergibt. Da clic Schraubel mit den fest daraufsitzenden Muffen dem ganzen Glied cilleı Halt geben wie ein ununterbrochener Gipsverband, so können die Verletzten in diesem transportiert werden. ja sie können sogar danit aufstehen und gchell.

Wann dicser Schraubenstreckverband angelegt werden kaun, wird sich als der Größe und Art der Bluchstïckverschiebung ergeben Nach dem Hackenbruchsohell Prinzip lassen sich auch bei den schwersten Knochenvelletzungen gute Heilungen ohıe Verkür/ully del Gliedulaßen crzieleı. Stehen die Distraktionsklammern nicht zul Verfinglulg, so konnen die von mir verwalldten Schrauben mit Muffell cinen einfuchell, billigen und doch wirksamen Elsatz bieten. 